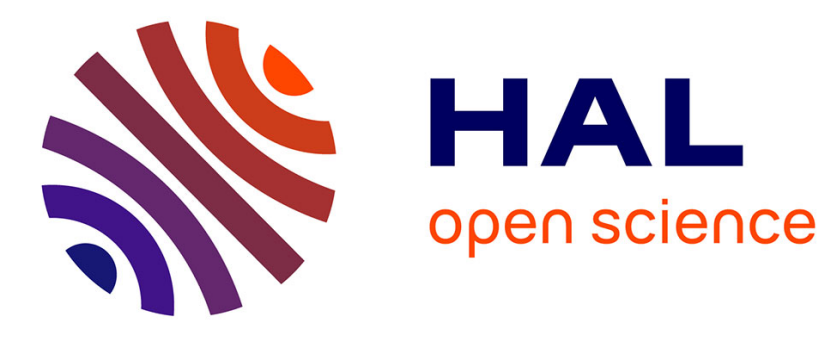

\title{
Gel-induced dew condensation
}

R. Urbina, S. Lefavrais, L. Royon, A. Mongruel, W. González-Viñas, D. Beysens

\section{To cite this version:}

R. Urbina, S. Lefavrais, L. Royon, A. Mongruel, W. González-Viñas, et al.. Gel-induced dew condensation. Journal of Hydrology, 2021, 599, pp.126263. 10.1016/j.jhydrol.2021.126263 . hal-03474563

\section{HAL Id: hal-03474563 https://hal.sorbonne-universite.fr/hal-03474563}

Submitted on 10 Dec 2021

HAL is a multi-disciplinary open access archive for the deposit and dissemination of scientific research documents, whether they are published or not. The documents may come from teaching and research institutions in France or abroad, or from public or private research centers.
L'archive ouverte pluridisciplinaire HAL, est destinée au dépôt et à la diffusion de documents scientifiques de niveau recherche, publiés ou non, émanant des établissements d'enseignement et de recherche français ou étrangers, des laboratoires publics ou privés. 
3 R. Urbina ${ }^{1,2}$, S. Lefavrais ${ }^{1,3}$, L. Royon $^{4}$, A. Mongruel $^{1}$, W. González-Viñas ${ }^{2}$, D. Beysens ${ }^{1,3, *}$

4

$5 \quad{ }^{1}$ Physique et Mécanique des Milieux Hétérogènes, UMR 7636 CNRS, ESPCI Paris - PSL

6 University,

7 Sorbonne Université, Sorbonne Paris Cité, 75005 Paris, France

$8 \quad{ }^{2}$ Universidad de Navarra, PHYSMED and Complex Systems groups, Pamplona. Spain

$9 \quad{ }^{3}$ OPUR, 2 rue Verderet, 75016 Paris, France

$10 \quad{ }^{4}$ Laboratoire des Énergies de Demain, Sorbonne Paris Cité, UMR 8236CNRS, 75013

11 Paris, France

$12 *$ Corresponding author E-mail: daniel.beysens@espci.fr

13

14 Keywords

Water Harvesting; Hydrogel; Dew Condensation; Vapor Adsorption

Highlights

- Hydrogels harvest more dew water from atmosphere than regular bare surfaces

- Dew enhancement on hydrogels is due to vapor adsorption adding to condensation

\section{Abstract}

Hydrogels are known to adsorb a large amount of vapor and liquid water, making them good candidates to enhance the amount of dew condensed from atmosphere. Although water vapor adsorption and liquid invasion in hydrogels have been the object of many studies, water condensation has been only little investigated. We address here the process of dew 
condensation on hydrogel grains widely used in agriculture (Aquasorb $3005^{\mathrm{TM}}$ ). We show that dew condensing on hydrogels is enhanced when compared to a regular bare substrate due to vapor adsorption, which adds to condensation. Hydrogels, which can both capture water by vapor adsorption and condense water vapor with high efficiency, are thus good candidates to harvest water vapor from atmosphere with higher yield than regular bare surfaces.

\section{Introduction}

Hydrogels are three-dimensional networks of hydrophilic polymers, whose properties are known since more than 50 years (see e.g. Wichterle and Lím, 1960). There are many types of hydrogels (e.g. pH-, temperature-, electro- sensitive, light-responsive, etc.), with several applications related to their extraordinary swelling properties (volume can be multiplied by 1000) when immersed in liquid water (for a review, see e.g. Majee, 2016; Ganji et al., 2010). The osmotic pressure attributed to the polymer network is the driving force of swelling. An osmotic gradient indeed forms between the water solvent, low in ionic solute, towards the polymer, rich in ionic solute. The swelling process distends the network and is counterbalanced by the elastic contractility of the stretched polymer network. Due to this osmotic pressure, hydrogels can also exhibit high water adsorption from water vapor (they are hygroscopic), an adsorption which increases with relative humidity $R H$ (see e.g. Delavoipière et al., 2018), making them suitable for atmospheric water harvesting (Zhao et al., 2019).

Water in hydrogels can be recovered by a moderate $(\sim 1$ bar $)$ osmotic mechanical pressure (Milimouk et al., 2001; Zhang et al., 2017). The osmotic pressure exerted by plant roots in presence of soil water, on the order of $0.1-1.2 \mathrm{MPa}$, can be high enough to extract water from the gel mixed with soil and so provide water to the plant roots (Rudzinski et al., 2002; Puoci et al., 2008). For agriculture in arid and semi-arid environments, there is an obvious 
interest to swell the gels with water obtained at the place where they need to be used, thus preventing water transportation on long distances. In consequence, collecting water from humid air by adsorption and/or dew condensation is very appealing.

The question we thus address in this study is whether, once exposed to humid air at night and cooled near or below the dew point, a typical hydrogel used in agriculture (Aquasorb $3005^{\mathrm{TM}}$ ) could adsorb and/or condense more water than regular surfaces do. It appears that hydrogel can indeed condense water at a larger rate than a bare substrate. In addition, when gel temperature is above the dew point temperature, water adsorption still occurs.

The paper is organized as follows. After this introduction, details about the experiments and methods are given. Then the water adsorption process is studied for relative humidity lower than $100 \%$, followed by condensation / adsorption proceeding at supersaturation larger than 1 . The paper ends by some concluding remarks concerning the main results of the study.

\section{Experiments and methods}

The absorbent materials are samples of Aquasorb $3005^{\mathrm{TM}}$, manufactured by SNF Floeger, a granular polyacrylamide. The materials are cross-linked copolymers of acrylamide and potassium acrylate, which are water insoluble and have a very high absorption capacity (ratio water weight/hydrogel weight up to 400). Further information can be found in Table 1 and from Aquasorb $3005^{\mathrm{TM}}$ (2020). The samples can be found in three sizes presentation (Dąbrowska and Lejcuś, 2012): small-size grains (diameter 0.19 $\pm 0.07 \mathrm{~mm}$ ), medium-size grains (diameter $0.70 \pm 0.2 \mathrm{~mm}$ ) and large-size grains (diameter $1.5 \pm 0.4 \mathrm{~mm}$ ). Hereby we report results with small and medium grains.

The experimental apparatus is depicted in Fig. 1. It is placed inside a climatic chamber where air temperature and humidity are controlled. The setup consists of a cooling device, a silicon 
(Si) plate of diameter $10 \mathrm{~cm}$ and thickness $0.7 \mathrm{~mm}$ (condensing surface $S_{c}=7.853 \times 10^{-3} \mathrm{~m}^{2}$ ), which holds the considered sample, and a balance to register the overall weight of sample and Si plate. The cooling device is held by a motorized support which moves it from an upper position, where it is in contact with the plate, to a lower position, where the plate is suspended by holders attached to the electronic balance and can be weighted. The Si plate is usually in contact with the cooling device. Each $38 \mathrm{~s}$, the plate is moved to the lower position to be weighed, a step which lasts about $8 \mathrm{~s}$. Temperature of the cooled plate is measured by calibrated thermocouples with an accuracy of $0.1^{\circ} \mathrm{C}$ and a sensitivity of $0.01^{\circ} \mathrm{C}$. In the chamber, air temperature $T_{a}$ is set at $20 \pm 0.5{ }^{\circ} \mathrm{C}$ and relative humidity at $50 \pm 3 \%$, which corresponds to a vapor pressure of $1.15 \mathrm{kPa}$ and a dew point temperature $T_{d}=9.3 \pm 0.1{ }^{\circ} \mathrm{C}$. The gel sample grains are poured on the silicon plate by the help of a circular mold which keeps the sample height less than $1 \mathrm{~mm}$ (small and medium grains) to form a thin layer. In order to have identical departure conditions in all cases, all the samples were previously dried at $40{ }^{\circ} \mathrm{C}$ during 8 hours and stored in a closed box with silica gel to maintain a low humidity level. For each experiment, the conditions inside the chamber and the cooling device temperature are fixed at their desired value before placing the plate with the gel.

Two different conditions were investigated, above or below the dew point, which corresponds to either relative humidity $R H \leq 100 \%$ (above the dew point) or supersaturation $S R>1$ (below the dew point). Relative humidity and supersaturation are defined from water vapor pressures as

$$
S R \text { or } R H / 100=\frac{p\left(T_{a}\right)}{p_{S}\left(T_{p}\right)}
$$

Here, $p\left(T_{a}\right)$ is the water vapor pressure in the air surrounding the plate and $p_{s}\left(T_{p}\right)$ is the water vapor saturation pressure at substrate (plate) temperature. In addition to the 
101 determination of characteristic adsorption isotherms under various $R H$, typical temperature conditions as encountered at night outdoors were specially investigated: (i) Above the dew

103 point with a plate temperature $T_{p}=T_{d}+4 \mathrm{~K}$ (adsorption), (ii) below the dew point : $T_{p}=T_{d}-4$

$104 \mathrm{~K}$.

105

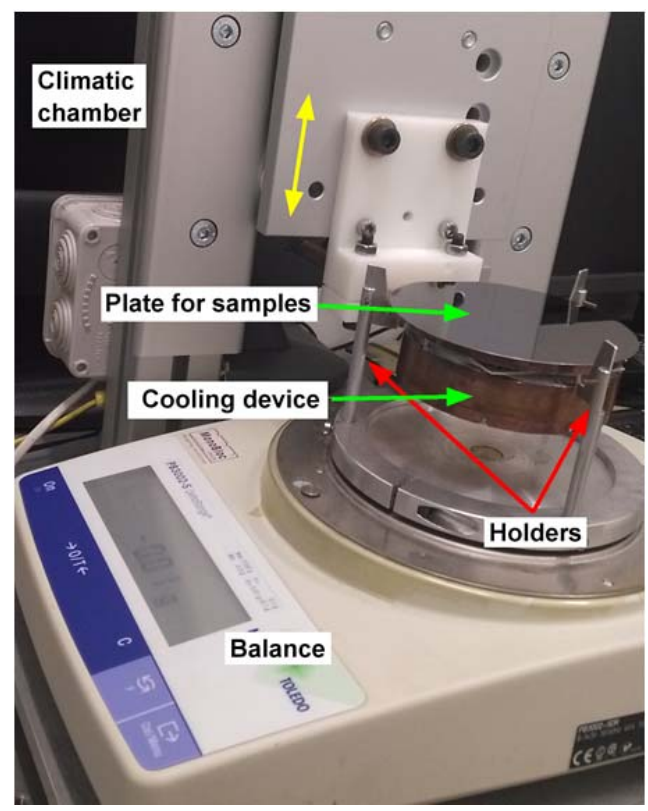

108 Fig. 1. Experimental setup in the climatic chamber. The sample is placed on a silicon plate

109 cooled from below. The cooling device moves vertically (yellow double arrow). In the upper

110 position, the cooling device is in contact with the plate and controls its temperature. In the

111 lower position, the plate remains suspended from the holders fixed to the balance (red arrows)

112 and the weight can be measured. A thermocouple is fixed below the plate to measure its

113 temperature $T_{p}$.

114

\begin{tabular}{ccc}
\hline Parameters & small size & medium size \\
\hline Grain diameter $(\mathrm{mm})$ & $0.19 \pm 0.07$ & $0.70 \pm 0.20$ \\
$\begin{array}{c}\text { Specific weight }\left(\mathrm{g} . \mathrm{cm}^{-3}\right) \\
\text { Absorption capacity in } 24 \mathrm{~h} . \\
\text { (water mass/dry gel mass) }\end{array}$ & 336 & 1.1 \\
\hline
\end{tabular}


118 Table 1. Some data on Aquasorb $3005^{\mathrm{TM}}$ gel (from Dąbrowska and Lejcuś, 2012 and 119 Aquasorb 3005, 2020).

\section{Above the dew point}

122 The dynamics of adsorption is classically governed by two processes (see e.g. Ganji et al.,

123 2010). One process is water molecules diffusing in the gel by Fick's law due to the difference

124 of water concentration between humid air ( $c_{0}$, corresponding to water vapor pressure $\left.p \sim R H\right)$

125 and water concentration in gel $\left(c_{c}\right)$. The other process is the stretching relaxation of the

126 polymer chains.

127 For typical times corresponding to adsorption amplitudes lower than $60 \%$ of the limiting

128 saturation value, the adsorption data measured by $\omega_{i}=m_{w} / m_{i}$ ( $m$ corresponds to mass with

129 subscript $w$ for water and subscript $i=s$ or $m$ for dry small and medium size gel grains) can

130 be fitted to the power law revealing the mode of relaxation:

$$
\omega_{i}=m_{w} / m_{i}=A_{i} t^{x}
$$

$134 A_{i}$ is an amplitude and $x$ an exponent whose value corresponds to the gel relaxation process.

135 For pure Fickian diffusion, $x=1 / 2$. For pure gel relaxation, $x=1$. In general, the exponent is

136 between $1 / 2$ and 1 , corresponding to both relaxation-controlled transport and diffusion-

137 controlled processes. Equation 2 can thus be generalized. With $A_{i}, B_{i}$ corresponding to

138 amplitudes related to the two processes:

139

$$
\omega_{i}=A_{i} t^{1 / 2}+B_{i} t
$$


142 For adsorption amplitudes larger than $60 \%$ of the limiting saturation value, the approach to

143 saturation can be described in the case of diffusion by the Fick's law. For a spherical particle

144 of radius $R$, initial radius $R_{\mathrm{i}}$ and surface $S=4 \pi R^{2}$ one obtains, neglecting the radius change

145 in the concentration gradient as swelling remains small in the adsorption process:

146

$$
\frac{1}{S} \frac{d m_{w}}{d t}=-D_{p} \nabla c=D_{p} \frac{c_{0}-c_{c}}{R} \sim D_{p} \frac{c_{0}-c_{c}}{R_{i}}
$$

149 where $D_{p}$ is the water diffusion coefficient in the polymer.

150 The adsorbed mass corresponds to water concentration in the gel, $c_{c}=3 m_{w} /\left(4 \pi R_{i}^{3}\right)$. The

151 solution to this equation leads to the following exponential decay evolution, in terms of ratio $152 \omega_{i}$ :

$$
\omega_{i}=\omega_{i, \infty}\left(1-e^{-t / \tau_{i}}\right)
$$

156 Here $\omega_{i, \infty}$ is the maximum rate of water in the saturated gel,

$$
\omega_{i, \infty}=\frac{4 \pi R_{i}^{3} c_{0}}{3 m_{i}} \propto R H
$$

160 and the typical relaxation time, $\tau_{i}$, reads as

161

$$
\tau_{i}=\frac{R_{i}^{2}}{3 D_{p}}
$$


164 Since the diffusion of vapor around the grains limits the process (as in the case of thin film

165 studies by Delavoipière et al. (2018)), the adsorption dynamics depends on the thickness of

166 the diffuse boundary layer, that is the layer where a Peclet number $P e=\frac{U L}{D}<1$. Here, $U$ is

167 the air flow velocity far from the substrate, $L$ is the characteristic length of the substrate and $D$

168 is the diffusivity of water molecules in air. In our study, the hydrodynamic conditions are the

169 same for all studied substrates, small or medium grains, and difference in kinetics can be only

170 attributed to $R H$ and the grain size.

171 In the case where the gel relaxation dominates the process, a relation similar to Eq. 5 is found,

172 however with a relaxation time which is independent of gel grain radius and relates to the

173 relaxation of the gel network,

174

175

176

177

178

179

180

181

182

184

185

186

187

$$
\omega_{i}=\omega_{i, \infty}^{\prime}\left(1-e^{-t / \tau_{i}^{\prime}}\right)
$$

with $\tau_{i}^{\prime}=$ const.

The evolution due to diffusion plus gel relaxation can thus be written as:

$$
\omega_{i}=\omega_{i, \infty}\left(1-e^{-t / \tau_{i}}\right)+\omega_{i, \infty}^{\prime}\left(1-e^{-t / \tau_{i}^{\prime}}\right)
$$

In Fig. 2 are reported typical adsorption isotherms and in Table 2 the results of the fits to Eqs.

2,3 using data in the range $\omega_{i}<0.6 \omega_{i, \infty}$. For small grains, the exponent of single power law, Eq. 2, are found in the range [0.58-0.79], with mean value 0.69 closer to 0.5 than to 1 , showing that vapor diffusion plays the major role in the relaxation process. This is corroborated with double power law fit, Eq. 3, where the amplitude of the $t^{1 / 2}$ term is about 10 times larger than the $t$ term. For medium grains, the relaxation is too long to obtain data close 
to saturation, then the fit to exponential decay Eq. 5 is made over all the data range, which gives an estimation of $\omega_{m, \infty}$. Data are then fitted to power laws in the range $\omega_{m}<0.6 \omega_{m, \infty}$.

190 The results of the fit to the exponential relaxation, Eq. 5, are reported in Table 2. According to 191 Eq. 5, the relaxation times $\tau_{m} / \tau_{s} \approx\left(R_{m} / R_{s}\right)^{2} \approx 13.6$. The measured ratio is smaller, $\tau_{m} / \tau_{s} \approx$ 1924.88 , confirming that gel relaxation also matters. Considering only water diffusion in the 193 polymer, one infers $D_{p} \sim 10^{-11} \mathrm{~m}^{2} \cdot \mathrm{s}^{-1}$, a typical value in gels.

194 Data (Fig. 2a) show, as expected (see e.g. Delavoipière et al., 2018), that $R H$ is the only 195 parameter for the adsorption amplitude $\omega_{i, \infty}$ (see the isotherms at $T_{a}=T_{p}=T_{d}+11 \mathrm{~K}$ and $T_{d}$ $196+4 \mathrm{~K}$ with air at same temperature than the substrate and $R H=78 \%$ ). Adsorption where the 197 plate temperature is at a temperature lower than $\operatorname{air}\left(T_{p}=T_{d}+4 \mathrm{~K}\right.$, air at $T_{a}=20^{\circ} \mathrm{C}$ and $R H=$ $19850 \%$ ) would correspond to a mean isotherm at $R H \approx 64 \%$. Medium grains isotherms (Fig. 2b) 199 correspond to the last case $\left(T_{p}=T_{d}+4 \mathrm{~K}\right.$, air at $T_{a}=20^{\circ} \mathrm{C}$ and $\left.R H=50 \%\right)$.
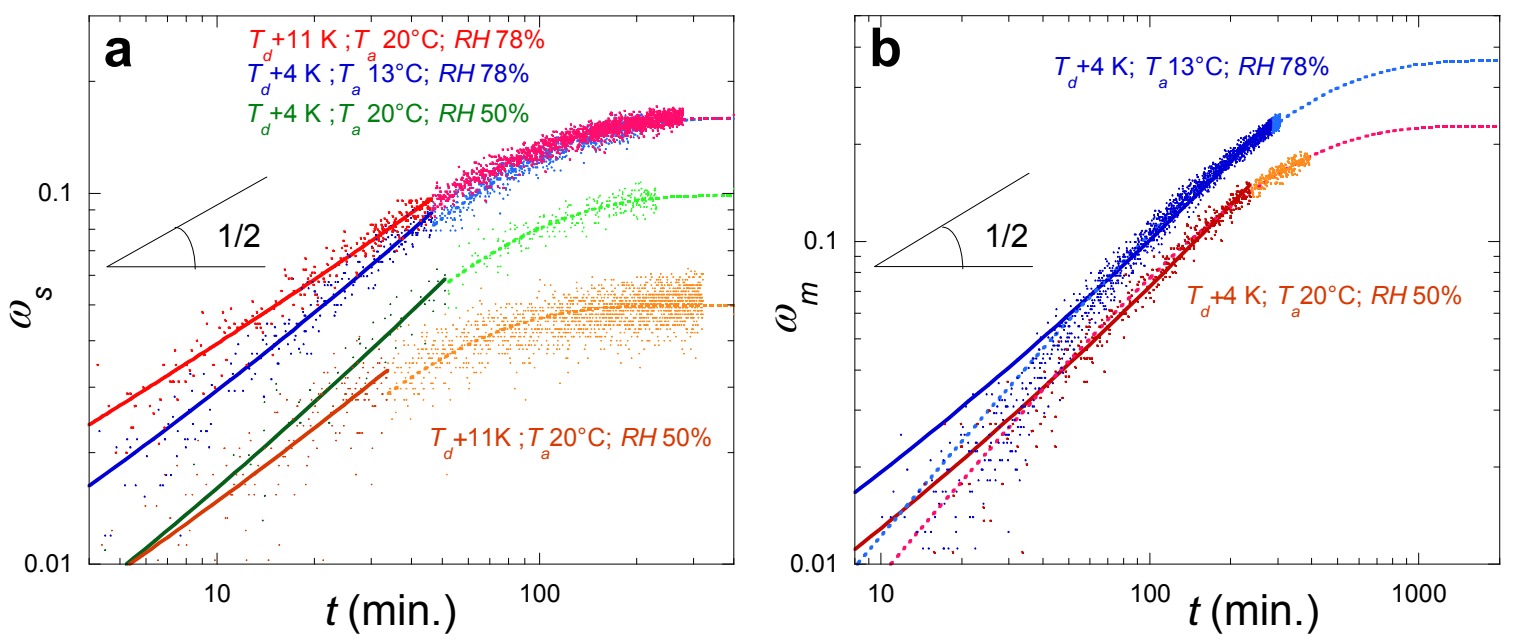

203 Fig. 2. Evolution of sorption isotherms $\left(\omega_{i=s, m}, \log -\log\right.$ plot, $\left.R H<100 \%\right)$. The full straight 204 lines are power law fits to data $60 \%$ below the saturation value (Eq. 2, darker points)) and the 205 interrupted curves are exponential fits to data above $60 \%$ of the saturation value (Eq. 5) (see 
Table 2). (a) Small grains. (b) Medium grains, where exponential fit is made with all data (see text).

\begin{tabular}{|c|c|c|c|c|c|c|c|c|c|}
\hline \multirow[t]{4}{*}{ Fitting } & \multirow{3}{*}{$\begin{array}{c}\text { Parameter } \\
R H(\%) \\
T_{p}-T_{d}(\mathrm{~K}) \\
\end{array}$} & \multicolumn{4}{|c|}{ Small grains } & \multicolumn{2}{|c|}{ Medium grains } & \multicolumn{2}{|c|}{ Bare } \\
\hline & & 50 & $\approx 64$ & \multicolumn{2}{|c|}{78} & $\approx 64$ & 78 & $S R$ & 1.32 \\
\hline & & 11 & 4 & 4 & 11 & 4 & 4 & & \\
\hline & $m_{i}(\mathrm{~g})$ & 9.75 & 9.14 & 9.20 & 9.00 & 7.34 & 7.14 & & \\
\hline \multirow{2}{*}{$\begin{array}{l}\text { Single } \\
\text { power } \\
\text { law } \\
\text { Eq. } 2\end{array}$} & $A_{i}$ & $\begin{array}{c}0.0032 \\
\pm 4 \times 10^{-4}\end{array}$ & $\begin{array}{c}0.0026 \\
\pm 4 \times 10^{-4}\end{array}$ & $\begin{array}{c}0.0056 \\
\pm 2 \times 10^{-4}\end{array}$ & $\begin{array}{c}0.0102 \\
\pm 2 \times 10^{-4}\end{array}$ & $\begin{array}{c}0.0169 \\
\pm 8 \times 10^{-5}\end{array}$ & $\begin{array}{l}0.00257 \\
\pm 5 \times 10^{-5}\end{array}$ & & \\
\hline & $x$ & $\begin{array}{c}0.66 \\
\pm 0.03\end{array}$ & $\begin{array}{c}0.79 \\
\pm 0.04\end{array}$ & $\begin{array}{c}0.72 \\
\pm 0.01\end{array}$ & $\begin{array}{c}0.58 \\
\pm 0.01\end{array}$ & $\begin{array}{c}0.81 \\
\pm 0.03\end{array}$ & $\begin{array}{c}0.80 \\
\pm 0.03\end{array}$ & & \\
\hline \multirow[t]{2}{*}{$\begin{array}{c}\text { Double } \\
\text { power } \\
\text { law } \\
\text { Eq. } 3\end{array}$} & $A_{i}$ & $\begin{array}{c}0.0034 \\
\pm 4 \times 10^{-4}\end{array}$ & $\begin{array}{c}0.0026 \\
\pm 6 \times 10^{-4}\end{array}$ & $\begin{array}{l}0.00608 \\
\pm 3 \times 10^{-4}\end{array}$ & $\begin{array}{c}0.0109 \\
\pm 2 \times 10^{-4}\end{array}$ & $\begin{array}{c}0.00423 \\
8 \\
\pm \underset{6}{6} \times 10^{-}\end{array}$ & $\begin{array}{c}0.0026 \\
\pm 2 \times 10^{-4}\end{array}$ & & \\
\hline & $B_{i}$ & $\begin{array}{l}0.00040 \\
\pm 8 \times 10^{-5}\end{array}$ & $\begin{array}{c}0.0008 \\
\pm 1 \times 10^{-4}\end{array}$ & $\begin{array}{l}0.00102 \\
\pm 5 \times 10^{-5}\end{array}$ & $\begin{array}{c}0.0048 \\
\pm 4 \times 10^{-5}\end{array}$ & $\begin{array}{c}0.00058 \\
6 \\
\pm 9 \times 10^{-5} \\
\end{array}$ & $\begin{array}{c}0.0004632 \\
4 \\
\pm 1 \times 10^{-5} \\
\end{array}$ & & \\
\hline \multirow{2}{*}{$\begin{array}{l}\text { Expo- } \\
\text { nential } \\
\text { decay } \\
\text { Eq. } 5\end{array}$} & $\omega_{i, \infty}$ & $\begin{array}{c}0.0499 \\
\pm 1 \times 10^{-4}\end{array}$ & $\begin{array}{c}0.0989 \\
\pm 7 \times 10^{-4}\end{array}$ & $\begin{array}{c}0.1593 \\
\pm 5 \times 10^{-4}\end{array}$ & $\begin{array}{c}0.1592 \\
\pm 3 \times 10^{-4}\end{array}$ & $\begin{array}{c}0.228 \\
\pm 2 \times 10^{-3}\end{array}$ & $\begin{array}{c}0.365 \\
\pm 3 \times 10^{-3}\end{array}$ & & \\
\hline & $\tau_{i}(\min )$. & $\begin{array}{c}39 \\
\pm 0.6\end{array}$ & $\begin{array}{c}60 \\
\pm 1.5\end{array}$ & $\begin{array}{l}64.8 \\
\pm 0.6\end{array}$ & $\begin{array}{l}55.8 \\
\pm 0.4\end{array}$ & $\begin{array}{l}242 \\
\pm 2\end{array}$ & $\begin{array}{l}294 \\
\pm 3\end{array}$ & & \\
\hline $\begin{array}{l}\text { Lin. evol. } \\
\text { Eq. } 11\end{array}$ & $\begin{array}{c}d h / d t \\
\left(\times 10^{-3}\right. \\
\left.\text { mm.min. } .^{-1}\right)\end{array}$ & & & & & & & & $\begin{array}{l}07 \\
10^{-3}\end{array}$ \\
\hline
\end{tabular}

Table 2. Results of the fits of the adsorption data. Uncertainties: One standard deviation.

\section{Below the dew point}

215 When the substrate temperature is set below the dew point temperature, condensation takes

216 place. Condensation on gels exhibits specific features when compared with condensation on a 217 bare surface.

\subsection{Region of inhibited condensation.}


In Fig. 3 is shown thin $(<1 \mathrm{~mm})$ layers of small and medium gel grains in a circle of radius $R_{g}$

$\sim 15 \mathrm{~mm}$ on a bare $\mathrm{Si}$ surface at $T_{p}=T_{d}-6.7 \mathrm{~K}$. Dropwise condensation is observed on the $\mathrm{Si}$

223 surface naturally coated with an oxidation layer whose contact angle with water is $\theta \approx 60^{\circ}$

224 (see e.g. Narhe et al., 2004).

225 The hygroscopic nature of the gel due to the osmotic pressure built-up by its hydrophilic sites induces enhanced adsorption of water vapor and thus lowers vapor pressure at the gel border, giving rise to a region of inhibited condensation (RIC) on the bare silicon substrate surrounding the gel sample, with width $\delta^{*}$ as defined in Fig. 3. This RIC is similar to the dry region around lyophilic patches during diethylene glycol condensation (Schafle et al., 2003)

230 or around a salty droplet (Guadarrama-Cetina et al., 2014).

231 To obtain the $\delta^{*}$ and $R g$ values shown in Fig. 4, the processing of the images includes 232 smoothing, contrast enhancement and border detection, which was performed by using the 233 Fiji ${ }^{\circledR}$ routines. Due to the irregular borders (see Fig. 3), the average distance between the gel 234 and the condensation pattern was determined within a small region.
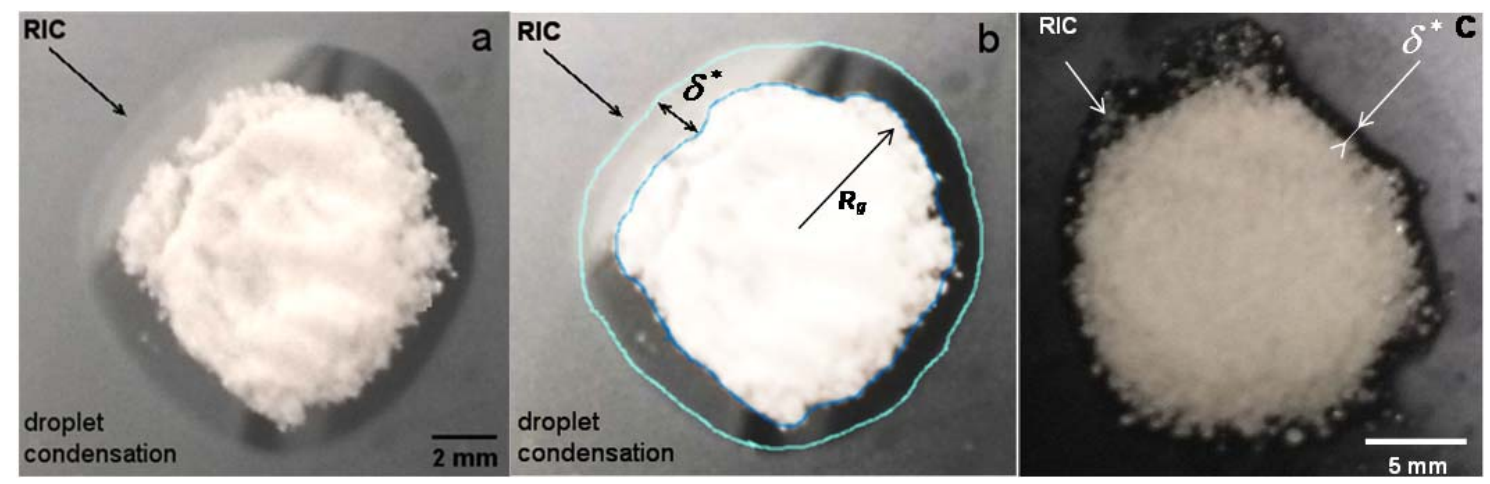

Fig. 3. Examples of region of inhibited condensation (RIC) around a thin $(<1 \mathrm{~mm})$ layer of (a, b) small size grain at $t=25 \mathrm{~s}$. (b) is the (a) picture with outlined gel and RIC contours. (c)

Medium size grains, $t=25 \mathrm{~s} .\left(T_{a}=22{ }^{\circ} \mathrm{C}\right.$ at $\left.47 \% \mathrm{RH}, T_{p}=T_{d}-6.7 \mathrm{~K}\right)$. 
243 Figure 4 reports the evolution of the RIC width $\delta^{*}$, which decreases with time. This RIC

244 decrease can be attributed to the increasing impregnation of the gel with adsorbed water.

245 When the gel is saturated, condensation on gel proceeds with the same way as on the bare 246 substrate, as seen in Section 4.2. The RIC width should be at that time the same as the RIC

247 observed around each water drops or hydrophilic dots (corresponding to the lyophilic patches 248 of Schafle et al., 2003), as a result of the surrounding water vapor gradient (see e.g. Beysens, 249 2018). The mechanism behind the RIC evolution is thus different to what happens with a 250 solute sample (e.g. a salty drop as in Guadarrama-Cetina et al., 2014), which comes from a 251 reduction of saturation pressure (Raoult law) or around an ice crystal where the constant drop 252 in saturation pressure makes the RIC width constant (Nath et al., 2018). (Further analysis is 253 out of the scope of the present study).

254 The evolution of the gel radius increase, $\Delta R_{g}=R_{g}(t)-R_{g}(0)$, is also shown in Fig. 3. The $255 \Delta R_{g}$ growth rate decreases after about a time $\sim 100 \mathrm{~s}$ for medium grains, a value near the time 256 where the condensation rate exhibits an inflection corresponding to the lowest influence of the 257 adsorption process (see Fig. 5). The $\Delta R_{g}$ increase is due to both adsorption and condensation 258 processes and is proportional to the collected increasing water volume per unit surface, $h$, 259 (see Eq. 11). The prefactor is, however, difficult to precisely evaluate because it depends on 260 the grains packing. It is clear in Fig. $4 \mathrm{~b}$ that the rate of growth is larger at early times, as in 261 Fig. 5. Note that the $\Delta R_{g}$ behavior can be affected by the grains rearrangement during their 262 swelling, which presumably explains the different behavior between small and medium grains 263 and the oscillations in the $R_{g}$ evolution as observed in Fig. $4 \mathrm{~b}$. 

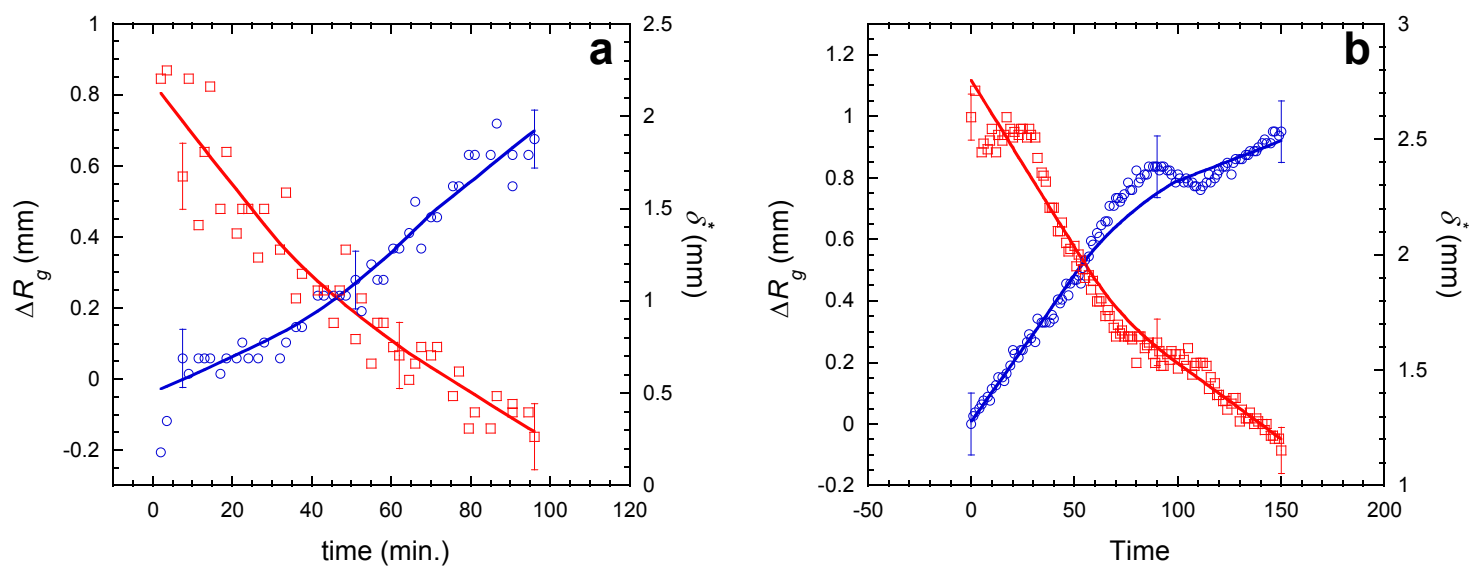

267 Fig. 4. Evolution of the RIC width $\delta^{*}$ (squares) and gel radius increase $\Delta R_{g}$ (circles) as

268 observed in Fig. 3 of (a) small grains and (b) medium grains. The curves are data smoothing.

$269\left(T_{a}=20^{\circ} \mathrm{C}, R H=50 \%, T_{p}=T_{d}-4 \mathrm{~K}\right)$.

270

4.2. Condensation rates

272

273 In order to compare the adsorption volume with respect to condensation on a bare substrate,

274 one considers the surfacic adsorbed volume or equivalent water film thickness, $h$. With $\rho$ the

275 water density, this layer is expected to grow proportionally to time as

276

277

$$
h=\frac{m_{w}}{\rho S_{c}} \sim t
$$




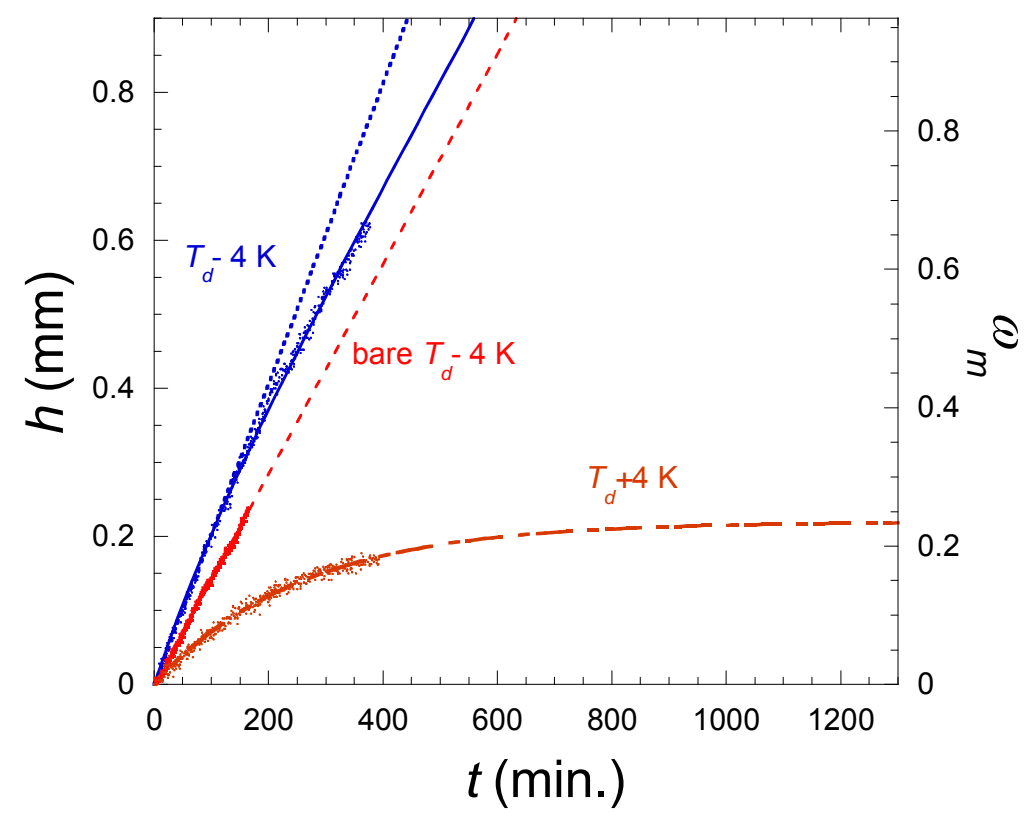

280 Fig. 5. Evolution of condensation $(S R=1.32)$ on gel medium grains (blue dots) and bare

281 substrate (red dots) under same conditions $\left(T_{a}=20^{\circ} \mathrm{C}, R H=50 \%, T_{p}=T_{d}-4 \mathrm{~K}\right)$. Left

282 ordinate: water volume per unit projected area, $h$. The continuous curve is a fit of gel data to 283 Eq. 12 and the interrupted line is a fit of bare substrate to Eq. 11. The dotted line is the initial 284 slope for condensation on gel. For the sake of comparison, the sorption isotherm at $T_{a}=20$ 285 ${ }^{\circ} \mathrm{C}, R H=50 \%, T_{p}=T_{d}+4 \mathrm{~K}$ is also reported (orange dots; right ordinate; see Fig. $2 \mathrm{~b}$ ).

In Fig. 5 is reported condensation on gels and bare substrate. The bare substrate condensation 288 is proportional to time with slope $(d h / d t)_{b}=1.4207 \times 10^{-3} \pm 2 \times 10^{-6} \mathrm{~mm} \cdot \mathrm{min}^{-1}$ (uncertainty: $1 \mathrm{SD})$. Gel exhibits a larger initial rate up to time $\approx 100 \mathrm{~min}$. where the rate progressively

290 decreases to reach the bare substrate value, corresponding to condensation and imbibition of 291 the gel as if it were a bare substrate. The swelling ratio at which this inflection occurs $(\sim 0.2)$

292 is far less than the maximum swelling ratio of $\sim 370$ and cannot be attributed to the vicinity of 293 this limit. One notes that further to this inflection the slope becomes comparable to what is 294 found on the bare surface. This rate decrease towards the bare substrate value can be related to 
the corresponding decrease of the RIC width and decrease of the rate of gel radius growth,

$296 \Delta R_{g}$, which occurs during the same time period, and which corresponds to the approach of the 297 saturation of adsorption. In order to assess this point, the gel data have been fitted to the sum 298 of condensation as if the gel were a bare substrate, Eq. 11 and the adsorption evolution, in 299 volume per unit surface as in Eq. 11:

300

$$
h=\left(\frac{d h}{d t}\right)_{b} t+h_{m, \infty}\left(1-e^{-t / \tau_{m}}\right)
$$

302

From Table 2, the amplitude value of the adsorption data at $T_{d}+4 \mathrm{~K}$ can be expressed in

volume per projected gel surface area, $h_{m, \infty}=\omega_{i, \infty} m_{i} / S_{c}=(0.201 \pm 0.002) \mathrm{mm}$. Figure 5 reports the condensation data on gel and bare substrate at $T_{d}-4 \mathrm{~K}$ and the adsorption data at $T_{d}+4 \mathrm{~K}$. Gel condensation data are fitted to Eq. 12 with $(d h / d t)_{b}$ free and imposed. The results are listed in Table 3. When all parameters are left free, $(d h / d t)_{b}$ is found somewhat smaller than on the bare substrate, presumably because of the lack of data at very long times. The adsorption time is found somewhat lower $(195 \mathrm{~s})$ that at $T_{d}+4 \mathrm{~K}(300 \mathrm{~s})$. The amplitude $(0.24$ $\mathrm{mm})$ compares relatively well with what is expected $(0.207 \mathrm{~mm})$. With $(d h / d t)_{b}$ imposed, the

311 typical adsorption time is 3 time smaller than expected and the adsorption amplitude is too 312 small $(0.106 \mathrm{~mm})$. When the adsorption time is fixed at the value at $T_{d}+4 \mathrm{~K}(\approx 300 \mathrm{~s})$, the amplitude $(0.165 \mathrm{~mm})$ increases but still remains smaller than the value at $T_{d}+4 \mathrm{~K}$. With the

314 latter amplitude imposed at $0.207 \mathrm{~mm}$ the adsorption time (391 s) becomes closer to the time 315 at $T_{d}+4 \mathrm{~K}(300 \mathrm{~s})$, with however a $\chi^{2}$ somewhat larger than the two previous fits. (A more 316 detailed analysis would need more data and is out of the scope of the present study). 


\begin{tabular}{|c|c|c|c|}
\hline$\chi^{2}$ Parameters & $\begin{array}{c}(d h / d t)_{b} \\
\left(\text { mm.min. }^{-1}\right)\end{array}$ & $\begin{array}{l}h_{m, \infty} \\
(m m)\end{array}$ & $\begin{array}{c}\tau_{m} \\
\text { (min.) }\end{array}$ \\
\hline 0.070 & $(1.1 \pm 0.1) \times 10^{-3}$ & $0.24 \pm 0.05$ & $195 \pm 31$ \\
\hline 0.054 & $\left(1.4207 \times 10^{-3}\right)$ & $0.106 \pm 0.001$ & $115 \pm 4$ \\
\hline 0.082 & $\left(1.4207 \times 10^{-3}\right)$ & $0.165 \pm 0.001$ & $(300)$ \\
\hline 0.15 & $\left(1.4207 \times 10^{-3}\right)$ & $(0.201)$ & $391 \pm 5$ \\
\hline
\end{tabular}

Table 3. Results of the fit to Eq. 11 of condensation data on gel (Fig. 5). The values under brackets are imposed in the fit. The gel mass is $m_{m}=6.94 \mathrm{~g}$. Notations: see text.

It thus results a net increase of water absorbed on gel when compared to the bare substrate under same conditions, with value on the order of $0.1 \mathrm{~mm}$. This behavior can be explained by the fact that two phenomena are present: (i) an initial adsorption of water vapor, giving a vapor concentration gradient above the surface of the gel larger than on the bare substrate, at

327 the origin of a RIC, followed by (ii) a steady condensation with the same concentration

328 gradient as found above the water drops on the bare substrate. Water will continue to condense on the gel until it reaches its maximum swelling. As a result of the initial large water absorption rate, water absorbed by the gel is thus found in greater amount than water 331 condensed on the bare plate.

332 Note that if instead of gel particles, one considers a gel film, one should obtain similar

333 enhancement as the adsorption phenomenon is not due to the macroscopic shape of the gel but

334 to the intrinsic properties of the polymer network. A gel film will then equally serve to condense water vapor and the swelling mechanism due to adsorption plus condensation will

336 be the same. The only difference would be the value of the exposed area to condensation,

337 which is smaller, then the condensation rate will be lower. The maximum swelling volume 338 capacity, proportional to the gel volume, will also be smaller. As a matter of fact, such gel 339 film condensation has already been reported by Delavoipière et al., 2018. On the other hand, 
if there were any non-water vapor absorbing hydrophilic powder on the surface instead of the

341 gel particles, one would simply expect no adsorption. Only filmwise condensation will occur

342 (coefficient $h_{m, \infty}=0$ in Eq. 12).

\section{5. Concluding Remarks}

346 Common hydrogels grains used for soil in agriculture (Aquasorb $3005^{\mathrm{TM}}$ ) can collect water

347 even for relative humidity less than $100 \%$. They also exhibit interesting properties when used

348 in supersaturation conditions (conditions of dew formation) since they collect more water than

349 a bare hydrophobic substrate with same projected surface area. The measured gain is on order

$350 \quad 0.1 \mathrm{~mm}$ of water / day, which is an important gain when compared to the current values found

351 for dew yield ( $0.1-0.6 \mathrm{~mm} /$ day $)$. This enhanced condensation of water is due to the initial

352 water vapor adsorption, which adds to condensation. As noted in the introduction, such

353 condensed water stored in gels can be either used in agriculture where the osmotic pressure

354 exerted by the roots is sufficiently high to extract water or removed by moderate $(\sim 1$ bar $)$

355 mechanical pressure for human use (Milimouk et al., 2001). Another way is to evaporate

356 water and condense it in e.g. a solar still (see e.g. Zhao et al., 2019 where details on needed

357 energy and time duration are given). Although the use is one and only in agriculture where the

358 grains are definitely mixed with the soil, water extraction by pressure or evaporation has to be

359 cyclically repeated. There is, however, a possible limitation to the number of cycles due to the

360 absorption of $\mathrm{CO}_{2}$ gas from the atmosphere. $\mathrm{HCO}_{3}{ }^{-}$ions are indeed added in the solutions,

361 screening the polymer charges and thus progressively reducing the swelling amplitude (see

362 e.g. Rička and Tanaka,1984 for ions influence). Precise evaluations remain to be carried out.

363 In addition, although the above study has been performed in laboratory by contact cooling,

364 preliminary experiments under outdoor radiative cooling (Beysens, 1998) give, at least 
qualitatively, the same results. Emissivity of dry gels is indeed high, as in any organic materials and, when wet, their emissivity becomes close to pure water emissivity ( 0.98 ; Schott et al., 2001). Outdoor radiative condensation unsurprisingly exhibits the same properties and constraints than condensation on a solid substrate (e.g. clear sky, high nocturnal relative

369 humidity, low wind speed, water evaporation under direct sun light; see Beysens, 2018).

370 Further research will be needed to get a Proof of Concept, but these results are already very 371 encouraging in view of the development of new materials to enhance dew water collection.

\section{Acknowledgments}

374

375 We gratefully thank E. Verneuil for discussions. R.U. acknowledges a financial support from 376 the "Asociación de Amigos de la Universidad de Navarra". This work was partially supported 377 by the Spanish AEI (Grants FIS2014-54101-P and FIS2017-83401-P).

\section{References}

Aquasorb $^{\text {TM }}$ 3005, 2020. https://snf.com.au/downloads/Aquasorb_E.pdf.

381 Beysens, D., 1998. Outdoor water adsorption and condensation on a polyelectolyte gel. CEA 382 Internal report.

383 Beysens, D., 2018. Dew Water. Rivers Publisher, Gistrup.

384 Dąbrowska, J., Lejcuś, K., 2012. Characteristics of selected properties of superabsorbents.

385 Polska Akademia Nauk 3/IV, 59-68. (In Polish). 
Delavoipière, J., Heurtefeu, B., Teisseire, J., Chateauminois, A., Tran, Y., Fermigier, M., Verneuil, E., 2018. Swelling Dynamics of Surface-Attached Hydrogel Thin Films in Vapor Flows. Langmuir 34, 15238-15244.

Ganji, F., Vasheghani-Farahani, S., Vasheghani-Faraahani, E., 2010. Theoretical Description 390 of Hydrogel Swelling: A Review. Iranian Polymer Journal (English) 5, 375 - 398.

Majee, S.B., (Ed.), 2016. Emerging concepts in analysis and applications of hydrogels ( 394 Intech, Rijeka).

Milimouk, I., Hecht, A.M., Beysens, D., Geissler, E., 2000. Swelling of neutralized 396 polyelectrolyte gels. Polymer 42, 487-494.

Narhe, R.D., Beysens, D.A., 2004, Nucleation and growth on a superhydrophobic grooved 398 surface. Phys. Rev. Lett. 93, 076103

399 Nath, S., Bisbano, C.E., Yue, P., Boreyko, J.B., 2018. Duelling dry zones around hygroscopic 400 droplets. J. Fluid Mech. 853, 601-620.

401 Puoci, F., Iemma, F. Spizzirri, U.G., Cirillo, G., Curcio, M, Picci, N., 2008. Polymer in 402 Agriculture: A Review. American Journal of Agricultural and Biological Sciences 3, 299-314.

403 Rudzinski, W.E., Dave, A.M., Vaishnav, U.H., Kumbar, S.G., Kulkarni, A.R. Aminabhavi, 404 T.M. 2002. Hydrogels as controlled release devices in agriculture: Review. Designed 405 Monomers and Polymers 5, 39-65.

406 Schäfle, C., Leiderer, P., Bechinger, C., 2003. Subpattern formation during condensation 407 processes on structured substrates. Europhys. Lett., 63, 394-400. 
408 Schott, J.R., Barsi, J.A., Nordgren, B.L., Raqueño, N.G., de Alwis, D., 2001. Calibration of

409 Landsat thermal data and application to water resource studies. Remote Sensing of

410 Environment 78, 108-117.

411 Wichterle, O., Lím, D., 1960. Hydrophilic Gels for Biological Use. Nature 185, 117-118.

412 Zhang Y.R., Tang, L.Q., Xie, B.X., Xu, K.J., Liu, Z.J., Liu, Y.P., Jiang, Z.Y., Dong, S.B., 413 2017. A Variable Mass Meso-Model for the Mechanical and Water-Expelled Behaviors of 414 PVA Hydrogel in Compression. International Journal of Applied Mechanics 9, 1750044.

415 Zhao, F., Zhou, X., Liu, Y., Shi, Y., Dai, Y., Guihua, Y., 2019. Super Moisture-Absorbent 416 Gels for All-Weather Atmospheric Water Harvesting. Adv. Mater., 31,1806446 